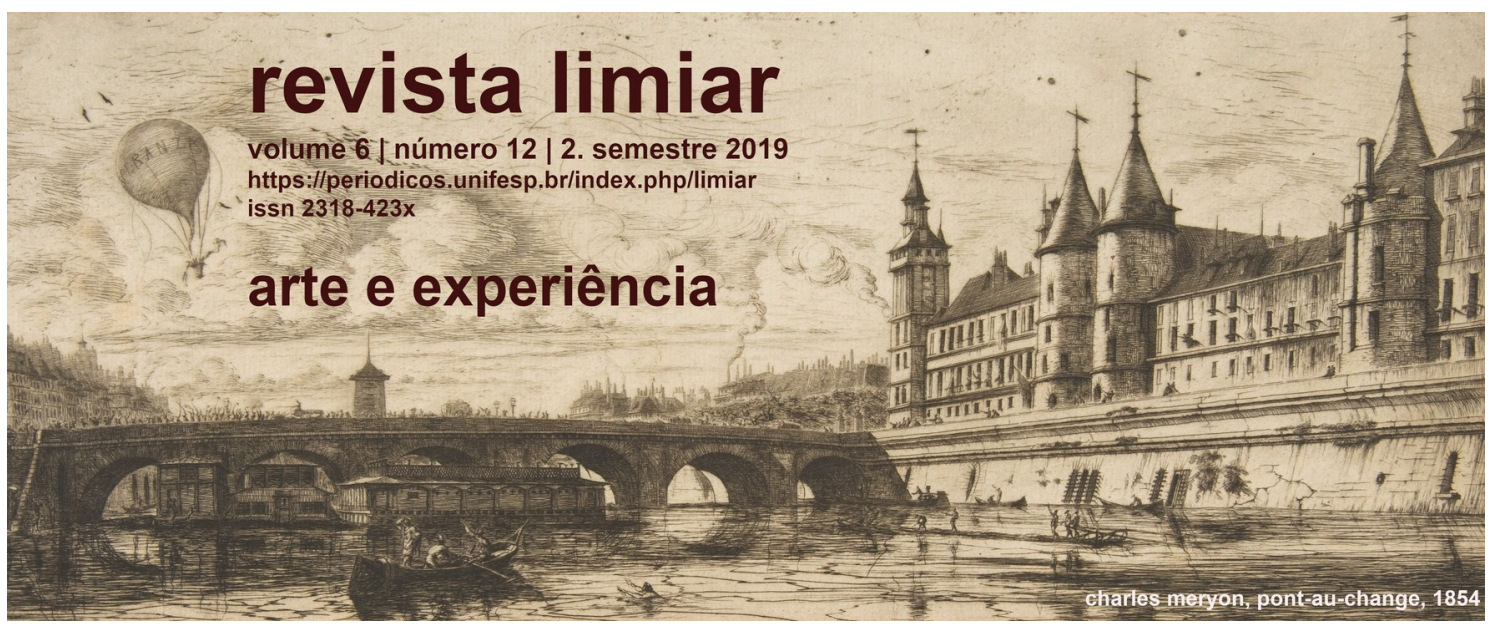

\title{
Mercado de arte e experiência artística no século XIX: o caso de Van Gogh e Millet
}

\author{
Felipe Sevilhano Martinez
}

Resumo: Este artigo estuda do mercado de arte do século XIX como um dos condicionantes da experiência artística de sua época. Trata-se de um mercado associado mais à personalidade artística do que às obras de arte, no qual, mais do que a obra em si, com seu tema e técnica, vende-se a carreira do artista que a criou. Nesse contexto, era comum a publicação de biografias associadas as principais artistas da segunda metade do século. Essas biografias tinham influência não só na maneira como o público se relacionava com as obras, mas também na percepção dos próprios artistas sobre sua atividade. É o caso de Vincent van Gogh, profundamente influenciado pela biografia de Jean-François Millet, escrita por Alfred Sensier dentro do contexto dos negócios do marchand francês Paul-Durand Ruel. O texto está dividido em três seções. A primeira delas apresenta as características gerais do mercado de arte na França da segunda metade do século XIX. A segunda detalha as práticas do marchand francês Paul Durand-Ruel, que incluem a publicação de biografias elogiosas aos artistas que vendia, no contexto da perda de importância da Academia e suas instituições. Finalmente, a terceira seção trata do impacto que a biografia de JeanFrançois Millet, publicada por Alfred Sensier em 1881, teve na formação da personalidade artística de Vincent van Gogh.

Palavras-chave: Mercado de arte, Van Gogh, Millet, Biografias, Durand-Ruel

Abstract: This paper addresses the art market as a central element of the artistic experience in the 19th-century. In this market biographies and personalities are more

* Doutorando em História da Arte e bacharel em Ciências Econômicas pela Unicamp. Atua como professor nos principais museus de São Paulo, como MAM e o MASP, onde também foi pesquisador bolsista. Realizou estágio doutoral no Museu Van Gogh, em Amsterdã, e no RKD - Nederlands Instituut voor Kunstgeschiedenis, em Haia. E-mail para contato: felipesmartinez@gmail.com 
important than the works of art. This means that the product to be sold is the artist and his temperament and not the painting - or the sculpture - with its theme and technique. Dealing in temperaments is also "dealing in biographies". For that reason, the publication of biographies of the most important artists of the time was very common. These biographies had an influence not only on the way the public related to the works of art but also on the perception of young artists about their own activities. This was the case of Vincent van Gogh, deeply influenced by the biography of Jean-François Millet, written by Alfred Sensier within the context of the business of the French art dealer Paul-Durand Ruel. The text is divided into three sections. The first presents the general characteristics of the art market in France in the second half of the nineteenth century. The second gives details of the practices of the French art dealer Paul Durand-Ruel, which includes the publication of biographies of the artists that he sold, in the context of the loss of importance of the Academy and its institutions. Finally, the third section addresses the impact that the biography of Jean-François Millet, written by Alfred Sensier in 1881, had on the formation of Vincent van Gogh's artistic personality.

Keywords: Art Market, Van Gogh, Millet, Biographies, Durand-Ruel

I

A pintura é um "depósito de uma relação social". Assim Michael Baxandall definiu as obras produzidas ao longo do Renascimento italiano. A partir dessa definição, descreveu os processos geradores dessa relação social ${ }^{1}$. Entre eles, estão os expedientes de produção e venda de obras de arte como parte da convenção representacional e da elaboração de um discurso capaz de guiar o olhar do espectador em relação à obra.

Com o mercado de arte da França do século XIX, berço da arte moderna, não foi diferente. As práticas de mercado forjadas no contexto da decadência do sistema de encomendas ligado à Academia e ao Estado ${ }^{2}$ tiveram influência não só na relação do público com as obras, mas também no modo com os artistas da época encaravam a si próprios. Um processo que se desenrola sob o quadro geral do desenvolvimento do capitalismo financeiro francês ${ }^{3}$, permeado por elementos de cidadania nascidos no

1 BAXANDALL, Michael. Painting and experience in fifteenth century Italy: a primer in the social history of pictorial style. New York, NY: Oxford University Press, 1988 p. 1-30.

2 A esse respeito sugiro a leitura de MAINARDI, Patrícia. The end of the salon: art and the state in the early Third Republic. Cambridge: Cambridge University Press, 1993.

3 A esse respeito, HARVEY, David. Paris, a capital da modernidade. São Paulo: Boitempo, 2015, pp. 161-171. 
contexto das Terceira República e da formação dos discursos modernos de individualidade ${ }^{4}$.

Mas se um pintor do quattrocento, como descrito por Baxandall, se defrontava com um sistema em que o patrono interferia em quase todo o processo produtivo inclusive na qualidade do pigmento $^{5}$-, o artista de meados do século XIX deverá vender sua produção diretamente ao mercado. É o artista quem deve propor ao público aquilo que será comprado ${ }^{6}$. Nesse ponto, há uma diferença importante entre o mecanismo de oferta e demanda que orienta a produção e a distribuição de pinturas nos dois momentos. No primeiro caso, o artista produz sabendo que há uma demanda definida, uma encomenda, muitas vezes estipulada em contrato; no segundo caso, o artista produz sem saber se sua oferta será efetivamente absorvida. Se no Renascimento, a demanda precede a oferta, na França do final do século XIX, a oferta precede a demanda ${ }^{7}$.

Nesse contexto, marchands como Paul Durand-Ruel foram responsáveis por interferir no mercado por meio de práticas como a formação de estoques, de lances artificiais em leilões e da promoção direta da arte que vendiam em revistas, catálogos de exposição e biografias. Mais que isso, conforme o século avança, esse mercado torna-se majoritariamente um mercado de paisagens. Primeiro, com os artistas da Escola de Barbizon e, ao final do século, com os impressionistas. As paisagens representavam não só a ascensão de um gênero considerado menor na Academia, mas também uma nova maneira de interpretar a relação entre artista e natureza. Elas eram o produto ideal para decorar os lares burgueses que aumentavam conforme o capitalismo francês se consolidava ${ }^{8}$. Mas esse novo mercado exigia um preparo do público.

4 Essas são as ideias centrais de GREEN, Nicholas; "Dealing in Temperaments: Economic Transformation of the Artistic Field in France during the Second Half of the Nineteenth Century". Art History, 10, março 1987.

5 BAXANDALL, op. cit. p.11.

6 Coli descreve a capacidade de Corubet de impor a seu público não somente o que deveria ser comprado, mas também a arte que deveria ser apreciada. Ver COLI, Jorge. O Corpo da Liberdade: reflexões sobre a pintura do século XIX. São Paulo/SP: Cosac Naify, 2010, p. 152.

7 Isso não significa que não houvesse pintores no Renascimento que vendessem para o mercado, nem pintores no final do século XIX que não aceitassem encomendas, naturalmente. A diferença está na proporção de cada modalidade em cada período: para um artista que quisesse construir uma reputação de sucesso no Renascimento, a via principal era o sistema de patronado, já para o artista do final do século XIX, era o mercado.

8 A esse respeito ver GREEN, Nicholas. The Spectacle of Nature: Landscape and Bourgeois Culture in Nineteenth-century France, Manchester University Press, 1990. 
O processo de educação do público para a arte moderna se dá pela construção de um aparato crítico e teórico por escritores como Zola, Phillipe Burty, Jules Castagnary e Esnest Chesneau. O entendimento se desloca de como interpretar um tema da tradição para a leitura de aspectos formais, como a pincelada e a sinceridade do contato entre o artista e a natureza. Saem de foco os temas da tradição; entra em cena a "natureza filtrada pelo temperamento", para citar a célebre frase de Zola tão mencionada por van Gogh em suas cartas ${ }^{9}$.

Desse modo, o mercado de arte do século XIX vende não somente obras de arte, mas também o temperamento dos artistas. Em primeiro lugar, vem a vida do artista, depois suas obras, de modo que vale mais ter "um Millet" ou "um Monet" do que uma paisagem concreta, com tema específico, feita por algum dos dois pintores. Em outras palavras, vende-se o indivíduo e não o produto de seu trabalho, mudança fundamental na compreensão da experiência artística da modernidade.

II

Com a exaltação da subjetividade dos artistas, tem início um processo de promoção e venda de personalidades - ou temperamentos - no lugar de obras individuais. Vender temperamentos também significa vender biografias. Não é por outra razão que a partir da década de 1870 uma série de biografias escritas sobre os paisagistas do meio do século começa a proliferar. Em 1870, Phillipe Burty tratou da vida de Paul Huet. Em 1872, Alfred Sensier publicou suas memórias sobre Théodore Rousseau; no ano seguinte, Frederic Henriet tratou de Antoine Chintreuil. Em 1875, foi a vez de Henri Dumensil tratar de Camille Corot, e em 1881, Louis Gonse escreveu sobre Eugène Fromentin ${ }^{10}$. Por fim, em 1885, Louis de Fourcaud escreveu sobre a vida de Bastien-Lepage ${ }^{11}$.

9 Cinthya e Harrison White, no seminal Canvases and Careers, Institutional Change in The French Painting World. Chicago: University Chicago Press, 1993 descrevem a existência de um "Dealer-Critic System" oposto ao antigo sistema de mecenato baseado na Academia e no Salão. Os autores assinalam que esse sistema estaria finalmente completo com 0 estabelecimento do impressionismo. Robert Jensen, no entanto, Marketing Modernism in Finde-Siècle Europe. Princeton: Princeton University Press, 1994, p.24, e Nicholas Green, op. cit., 1987, mostram que bem antes do impressionismo, já existem práticas que articulam o interesse de marchands e críticos.

10 Eugène-Samuel-Auguste Fromentin (1820-1876) além de pintor era também crítico. Participou duplamente do processo de venda de biografias. Tanto como biógrafo quanto como biografado.

11 Esses escritores não se limitavam a escrever biografias e atuavam em jornais, revistas e catálogos de exposição. Nem as biografias se resumem às aqui mencionadas, naturalmente 
Em alguns casos, essas biografias estavam diretamente ligadas aos interesses comerciais de seus autores. Veja-se o caso de Sensier que, além de escrever sobre Rousseau, também tratou de Georges Michel e de Jean-François Millet. Sobre este, escreveu uma biografia chamada "La Vie et l'Oeuvre de Jean-François Millet", fundamental na construção do ideal de artista para Van Gogh $^{12}$, como veremos a seguir. Além disso, Sensier tinha uma preciosa coleção de obras de artistas de Barbizon, parte da qual foi vendida a Durand-Ruel: ao longo da década de 1870, o marchand adquiriu 167 obras da coleção do escritor, entre desenhos e pinturas, o que incluía 34 pinturas de Millet e 9 de Rousseau ${ }^{13}$. Naturalmente, para Sensier, promover a vida de Millet, Rousseau e companhia era também promover seus próprios interesses materiais ${ }^{14}$.

As paisagens dos artistas da Escola de Barbizon possuíam, ao mesmo tempo, um caráter particular e coletivo. Em termos de vendas, essa era uma característica valiosa, já que podiam ser vendidas em sua individualidade e ao mesmo tempo identificadas com uma marca comum ao grupo. Além disso, já havia um terreno preparado para quando essas paisagens chegassem; banqueiros e industriais franceses possuíam grandes coleções de arte holandesa, notadamente paisagens, e artigos sobre suas coleções eram escritos com frequência por críticos como ThoréBüguer e Eugène Fromentin. Este escreveu, em 1876, um capítulo em seu Les Maîtres d'Autrefois sobre a influência da paisagem holandesa do século 17 na paisagem francesa, com especial ênfase na vida de Jacob van Ruysdael, cuja presença nas coleções de empresários e financistas do período, com os irmãos Pèreires, era frequente ${ }^{15}$.

pelo menos até os escritos de nomes como Fustel de Coulanges ou Gabriel Monod a história da arte é feita por jornalistas, críticos ou escritores não necessariamente comprometidos com métodos rigorosos. Por exemplo, críticos como Phillipe Burty variavam sua atividade jornalística e acadêmica. As biografias catapultavam a vida dos artistas, em uma tradição vasariana que exaltava a subjetividade privilegiada dos criadores com a combinação de informações factuais e anedotas. Ver Green, Nicholas; op. cit. 1987.

12 SENSIER, Alfred. La Vie et le oeuvre de Jean-François Millet. Manuscrit publié par Paul Mantz avec de nombreuses illustrations, Paris A. Quantin, Imprimeur-editeur, 7, Rue SaintBenoit. Disponível em https://gallica.bnf.fr/ark:/12148/bpt6k96825069.textelmage.

13 JENSEN, Robert.op. cit.. p.53.

14 Para efeito de comparação, em 1875, após a morte do artista, o arquiteto Gavet vendeu sua coleção de 95 pastéis de Millet por 637,450 francos, média de 6710 francos por obra. Claramente o marchand fez um bom negócio anteriormente ao comprar a coleção de Sensier. 15 Ver BOIME, Albert. Albert. "Entrepreneurial Patronage in Nineteenth-Century France", in Enterprise and Entrepreneurs in Nineteenth and Twentieth-Century France, ed. CARTER, Edward et al. (Baltimore and London: Johns Hopkins University Press, 1976), 331-51. 
Nicholas Green argumenta que a importância da subjetividade criadora é potencializada com a formulação de um novo esquema vindo de elementos surgidos na Terceira República. Entre eles está o contato do indivíduo com a natureza, que teve sua compreensão modificada a partir de termos criados na psicologia experimental, como "expressão", "percepção", ou "sensação", comuns nas biografias dos artistas do período, e pela própria ampliação do conceito de natureza, que deixou de levar em conta apenas a relação entre o artista e a paisagem, para considerar a relação do indivíduo com o mundo. Ainda segundo o autor, colaboram com essa mudança elementos de cidadania ligados ao positivismo e à crescente celebração de artistas paisagistas nas exposições universais ocorridas entre 1889 e 1890. Essas condições fertilizam o solo no qual biógrafos plantavam suas ideias acerca da importância individualismo criador $^{16}$.

Mas não eram somente artistas que tinham sua subjetividade exaltada. $O$ mesmo acontecia com colecionadores e marchands. Vejamos o caso do financista e colecionador Charles Edwards, caracterizado por um certo Jean Ravenal em um artigo da Revue International de l'Art e de la Curiosité, de 1870, como alguém que "realmente sabia apreciar a arte que comprava"17". A coleção de Edwards era repleta de obras de pintores como Millet e Rousseau, cujas pinturas foram preciosamente analisadas no artigo de Ravenal. Acontece que Jean Ravenal era ninguém menos do que Alfred Sensier, que sob esse pseudônimo escrevia textos elogiosos aos artistas vendidos por Durand-Ruel. A Révue International de l'art e de la curiosité, por sua vez, era uma publicação ${ }^{18}$ fundada pelo próprio marchand em 1869 com o propósito de promover seus artistas no mercado ${ }^{19}$. Mais que isso: Charles Edwards era o financista que forneceu o capital para Durand-Ruel realizar a maior parte de suas transações na

16 Esses elementos surgem da "articulação da personalidade criadora dentro do discurso artístico como resultado de uma mudança historicamente específica na organização do conhecimento, sustentada de um lado por condições internas - a transparência tradicionalmente atribuída à pintura de natureza, o "pull" institucional entre a literatura de entretenimento e a ciência profissional - e de outro lado pelas forças ideológicas dominantes moldando a cidadania na terceira república". O jornalismo e a expertise histórica garantem o valor cultural e de investimento da obra de arte. Ver GREEN, Nicholas. op. cit. 1987.

17 Ver ZAROBEL, John. Durand-Ruel and the Market for Modern Art, from 1870 to 1873. in "Paul Durand-Ruel and the Modern Art Market: Inventing Impressionism, National Gallery Company, London, Yale University Press, 2015 p. 65.

18 A Révue International duraria pouco, até 1871. Cerca de 20 Anos depois, Durand-Ruel repetiria o procedimento em com a Revue l'Art dans les deux mondes.

19 Em 1881, uma nova venda de obras da coleção do Edwards apresentava a coleção como "construída ao longo dos anos com grande finesse". Nela, as obras de Rousseau que tinham sido vendidas em 1870 custavam três vezes mais. Ver GREEN, op. cit, 1987. 
década de 1870, tendo como colateral as pinturas de sua galeria. Em outras palavras, Sensier, Edwards e Durand-Ruel eram parte de um mesmo grupo que tinha como fim valorizar artistas que trariam lucro aos três.

Desde 1860, Durand-Ruel empreendeu um esforço de monopolizar as obras dos artistas de Barbizon com o capital de Edwards. Por exemplo, em companhia do marchand Hector Brame, havia comprado boa parte da obra de Theodore Rousseau, morto em dezembro de 1867. Em seu leilão póstumo, a dupla arrematou 79 obras pela bagatela de 70 mil francos, e as adicionaram ao estoque já existente de 140 , entre pinturas e desenhos ${ }^{20}$. A partir de então, o marchand passou a quase monopolizar as obras disponíveis no mercado, o que naturalmente deu a ele considerável poder sobre os preços do artista. Edwards ganhava duplamente: com as taxas de juros que cobrava do marchand e com a valorização das obras de sua coleção.

Durand-Ruel também se esforçou para construir um estoque de obras de Millet. A tarefa não foi fácil. Em 1860, o marchand belga Arthur Stevens havia firmado um contrato de exclusividade com Millet por um prazo de três anos, que incluía todos os desenhos e pinturas produzidos no período. Antes que o contrato terminasse, entretanto, os preços de Millet haviam subido com tanta velocidade que o artista fez o que pôde para terminar o acordo e voltar a ter poder sobre sua produção. Sem sucesso. Quando finalmente o contrato expirou, Durand-Ruel e Brame foram rápidos ao se aproximar do artista, propondo a extravagante soma de 30 mil francos por ano em troca de toda sua produção. O pintor, prevenido pelos acontecimentos com Stevens, declinou. Mesmo assim, a dupla buscou comprar pinturas de Millet distribuídas pelas coleções de tal modo que no começo da década de 1870 quase monopolizavam a obra do artista ${ }^{21}$.

Como informa Jansen, em 1872, estavam sob a posse de Durand-Ruel obras como "Édipo derrubado de uma árvore" (National Gallery Ottawa), "A morte e o lenhador" (Ny Calrsberg Glytothek, Copenhage), "Pastor Sentado" (Boston Fine Arts Museum). No mesmo ano, o marchand vendeu "Redil de ovelhas sob a luz da lua" (Walter Arts Museum) por 40.000 francos, o dobro do valor que havia gasto meses antes; na mesma ocasião, também vendeu o Angelus (Museu d'Orsay) de Millet por 38000 francos. Exibiu o "Semeador" da coleção de Sensier de quem a comprou e vendeu no começo da década de 1880 por 12000 francos ao colecionador inglês

20 JENSEN, Robert op cit. p 23.

$21 \mathrm{lbid}$. 
William $\mathrm{H}$. Vanderbilt. Fica claro que as obras de Millet eram um ótimo negócio e que seu movimento de mercado contribui ainda mais para sua valorização. O ápice dessa valorização ocorreu ao final do século quando, em 1889, o marchand americano James Sutton comprou a obra Angelus na venda da coleção do industrial Eugène Sécretan por 553.000 francos, recorde de preços em um leilão, superando Antonin Proust, que representava o Louvre. No mesmo ano, o colecionador francês Hippolyte François Alfred Chauchard recomprou a pintura por 775.000 francos, presente em sua coleção até 1909 (data de sua morte), quando então é doada ao Louvre. O valor mais alto pago por uma obra de arte. A mesma venda em que Sutton arrematou a obra disponibilizava desenhos de Millet por 25000 francos, valores ainda muito acima dos sonhos da maioria dos impressionistas ${ }^{22}$.

O fato de Chauchard ter comprado a pintura de um americano também indica um movimento de repatriação de certas obras por colecionadores franceses ${ }^{23}$. Esse, naturalmente, é um fator que influencia diretamente o mercado de tais artistas. Em parte, os preços das obras dos artistas da Escola de Barbizon dispararam na segunda metade da década de 1870 porque uma parcela considerável delas era comprada por colecionadores americanos, saindo do mercado francês. As pinturas que cruzavam o atlântico terminavam em algum museu dos Estados Unidos, ou mesmo incorporadas a uma grande coleção sem pretensões de revenda. Com o mercado de paisagistas, abre-se o caminho para práticas comerciais que serão reeditadas por Durand-Ruel com os impressionistas anos depois.

III

Os artistas da Escola de Barbizon, que nas décadas de 1850 e 60 podiam ser comprados por valores modestos, ao final do século alcançavam preços estratosféricos. Conforme os preços aumentam, lucrar no mercado desses artistas torna-se cada vez mais difícil. Isso se explica pela redução na oferta gerada pelo fluxo de obras que atravessavam o Atlântico rumo ao mercado americano sem retornar, e pela morte dos principais artistas do grupo conforme o século avançava. Baixa oferta e alta procura fazem os preços disparar. Em dezembro de 1883, em carta a seu irmão,

22 ibid. p.53.

$23 \mathrm{lbid}$, p. 76. 
Vincent van Gogh percebe o potencial especulativo desse mercado e o compara à bolha das tulipas de Amsterdã do século XVII"24:

\begin{abstract}
Em relativamente poucos anos, o comércio de arte se desenvolveu a despeito da própria arte. Mas se tornou muito uma especulação de banqueiros e assim ainda é agora. Eu não diria inteiramente, mas diria muito. Porque na medida em que é uma bolha de empresa, não deveria ter o mesmo fim, do que digamos, a bolha de tulipas?(...)

Mas eu responderia que muitos ricos que compram pinturas caras por essa ou aquela razão não o fazem pelo valor artístico que veem nelas - a diferença entre tulipas e pinturas que você e eu vemos não é visível para eles - eles, especuladores e pochards blasés e um monte de outros, também comprariam tulipas agora, assim como no passado, se tivesse certa "chiqueza" nisso (...) Coisas que agora são muito altas em preço pode cair -. Você me pergunta: podem Millet e Corot cair, eu digo, sim EM PREÇO.

De qualquer modo, de um ponto de vista artístico Millet é Millet, Corot Corot, fixos, como o próprio sol, aos meus olhos.
\end{abstract}

Segundo van Gogh, os preços, sujeitos a variações e aos caprichos do mercado, não estariam conectados ao "real" valor da obra de arte, dado em termos puramente artísticos. E mais, o pintor ainda compara o mercado de arte e seu caráter volátil com as tulipas da Era de Ouro holandesa. Essa comparação também surge em outras 4 cartas, escritas em momentos diferentes da vida do artista ${ }^{25}$. Em todas a mensagem é clara: a especulação passa e a arte fica, o que reafirma a separação entre valor artístico e valor comercial, de natureza especulativa. A pergunta a ser feita é: um existiria sem o outro? É possível separar totalmente o valor artístico do valor de mercado, quando as próprias práticas de marchands como Durand-Ruel são responsáveis por consolidar as carreiras dos artistas e mesmo por legitimar critérios estéticos? A resposta é não: uma coisa não pode ser separada da outra.

Isso dito, há algo comum entre as pinturas de Millet, Rousseau, Bouguereau ou Monet para além das tintas e das telas, uma equivalência que as iguala a todas as

24 Carta 409, disponível no site www.vangoghletters.org. Original do trecho traduzido por mim: In betrekkelijk korte jaren ontwikkelde zich al wat kunsthandel is in rapport met de kunst zelf.Maar het werd maar al te zeer een soort bankiersspeculatie en is het nu ook - ik zeg niet geheel en al - ik zeg eenvoudig veel te veel. waarom, voor zoover 't windhandel is, zal 't er niet mee gaan als b.v. met den tulpenhandel? (...) Maar ik beweer dat veel rijke lui die om een of andere reden de dure schilderijen koopen het niet om de kunstwaarde doen die ze er in zien - voor hun is 't onderscheid dat gij en ik zien tusschen tulp en schilderij niet zigtbaar - zij, de speculateurs en pochards blasés en een boel anderen, zouden ook nu tulpen koopen, net als vroeger, als er maar een zekere chic in was.-Dingen nu hoog opgevoerd in prijs, kunnen zakken.- Vraagt ge mij, kan Millet en Corot zakken. zeg ik, ja IN PRIJS. Overigens van uit een artistiek oogpunt is Millet Millet, Corot Corot, vast - als de zon zelf, in mijn oog.

25 Cartas 527, 537, 626 e 811, disponíveis no site www.vangoghletters.org. 
demais mercadorias de sua época, como as tulipas de Van Gogh, espécie de valor de troca pelo qual podem ser negociadas e transformadas em objetos de especulação. Esse valor de troca, não necessariamente relacionado a seu valor artístico, também é parte de como a obra de arte aparece no século XIX. Em outras palavras, não é possível olhar para essas obras sem se ter em conta seu percurso no mundo financeiro; elas têm por isso mesmo um valor imaterial, necessariamente vinculado a sua capacidade de se valorizar. Seu percurso como mercadoria também é capaz de alterar a maneira como a olhamos. Um valor artístico diretamente influenciado pelas atividades de marchands e críticos.

Mas o que faz com que uma pintura de Millet possa valer 50 mil francos a mais ou a menos? Van Gogh diz que o valor de Millet é para ele tão constante quanto o sol, mas o próprio pintor holandês formou sua admiração por Millet, em grande medida, inspirado pelas ideias presentes na biografia de Sensier, que serviu para valorizar a coleção do próprio autor e de Durand-Ruel. Ou seja, parte daquilo que fez van Gogh crer que o valor artístico de Millet é tão sólido quanto o sol vem de expedientes comerciais que tinham como finalidade o aumento de preços. Mais que isso, a própria subjetividade do artista, um dos exemplos mais bem-acabados de artistas da modernidade, foi forjada a partir da biografia de Sensier.

Vejamos: o livro de Sensier foi publicado em 1881; já no ano seguinte, van Gogh leu suas primeiras páginas: foi uma espécie de choque de reconhecimento ${ }^{26}$, que trazia "coragem ${ }^{27 "}$ ao pintor que, com quase 30 anos, dava seus primeiros passos como artista. O Millet de Sensier materializava perfeitamente os ideais artísticos do pintor holandês em seus primeiros anos: recusava o meio urbano e encontrava uma espécie de verdade nos trabalhos e modos de vida tradicionais do campo. Tudo o que van Gogh esperava de um verdadeiro artista. Mais do que uma referência técnica, Millet foi uma referência biográfica, algo semelhante ao que o próprio pintor holandês será aos artistas do começo do século XX, notadamente os expressionistas.

Assim, Sensier construiu a imagem de Millet como um camponês desinteressado, para quem a atividade artística literalmente brotou em meio aos trabalhos de cultivo. A partir disso, o trabalho artístico se aliava perfeitamente ao trabalho do campo, espécie de reserva de estabilidade em um mundo onde tudo o que

26 Tilborgh, Louis van. et al Millet, Van Gogh: Paris Museé d'Orsay Paris: Réunion des musées nationaux: Diffusion, Seuil, 1998. p.11.

27 Carta 258, disponível em www.vangoghletters.org. 
era sólido desmanchava no ar. Uma associação perfeita para van Gogh, que ficava contente quando era confundido com um trabalhador e que se retratou com seu macacão azul de camponês por diversas vezes ${ }^{28}$. Além disso, Sensier aponta que Millet foi reconhecido somente após sua morte: algo não exatamente verdadeiro. Ainda que seu sucesso em vida não possa ser comparado àquele depois de sua morte, Millet foi reconhecido por seus pares e considerado um artista de talento mesmo por aqueles que discordavam de suas escolhas estéticas. Assim, de uma maneira exagerada, a trajetória do artista francês, tal qual desenhado na biografia, orienta as justificativas que Vincent dava a si próprio sobre seus próprios fracassos ao longo da carreira ${ }^{29}$.

É claro que biografia de Sensier trazia generalizações e exageros ${ }^{30}$. Elas, entretanto, não impediram que van Gogh, logo após ter terminado a biografia, concluísse que "a arte é um combate", acrescentando que após ter lido as palavras de Millet sentiu "a necessidade de rabiscar com um duro lápis de carpinteiro, em vez de um pincel macio ${ }^{31 "}$. Desse modo, tal qual o Millet de Sensier, quanto mais Vincent se aproximava de seu ideal de trabalhador, mais artista se tornava.

Mas se Sensier era um dos campeões de Durand-Ruel, no contexto das biografias que prosperam a partir da segunda metade do século XIX, é impossível pensar a imagem de artista que van Gogh construiu para si próprio - o que inclui a relação com os trabalhadores, a valorização da vida simples do campo e a rejeição às convenções - fora dessa economia da arte. Se o mercado de arte estava organizado mais em torno das subjetividades do que das obras, o exemplo de van Gogh e Millet mostra que o próprio mercado é também um dos condicionantes da experiência artística não só para o púbico, mas também para os próprios artistas. Em outras palavras, as posições de van Gogh em relação à arte de seu tempo não podem ser compreendidas fora do contexto das relações materiais da época. Assim, mais do que somente uma maneira de produzir e vender obras de arte, o mercado é um elemento central na experiência artística da modernidade.

28 Por exemplo, no "Autorretrato como pintor", presente no Museu Van Gogh; ou mesmo no "Passeio ao Crepúsculo", no acervo do MASP.

29 TILBORGH et al, op cit. p. 12.

30 Ibid, p. 11-15 e UITERT, Evert van, Vincent van Gogh in creative competition: Four essays from Simiolus, (dissertation Amsterdam) Zutphen 1983, p. 47-50.

31 Carta 210, disponível em www.vangoghletters.org. 


\section{Bibliografia}

BAXANDALL, Michael. Painting and experience in fifteenth century Italy: a primer in the social history of pictorial style. New York, NY: Oxford University Press, 1988.

BOIME, Albert. "Entrepreneurial Patronage in Nineteenth-Century France", in Enterprise and Entrepreneurs in Nineteenth and Twentieth-Century France, ed. CARTER, Edward et al. (Baltimore and London: Johns Hopkins University Press, 1976).

COLI, Jorge. O Corpo da Liberdade: reflexões sobre a pintura do século XIX. São Paulo/SP: Cosac Naify, 2010.

DEWEY, John. Arte como experiência. São Paulo. SP: Martins Fontes, 2010.

UITERT, Evert van. Vincent van Gogh in creative competition: Four essays from Simiolus. (dissertation Amsterdam) Zutphen 1983.

GREEN, Nicholas. "Circuits of Production, Circuits of Consumption: The Case of MidNineteenth-Century French Art Dealing", Art Journal, Vol. 48, No. 1, NineteenthCentury French Art Institutions (Spring, 1989).

The Spectacle of Nature: Landscape and Bourgeois Culture in Nineteenthcentury France. Manchester University Press, 1990.

"Dealing in Temperaments: Economic Transformation of the Artistic Field in France during the Second Half of the Nineteenth Century". Art History 10, março 1987. HARVEY, David. Paris, a capital da modernidade. São Paulo: Boitempo, 2015.

JENSEN, Robert. Marketing Modernism in Fin-de-Siècle Europe. Princeton: Princeton University Press, 1994.

MAINARDI, Patricia. The end of the salon: art and the state in the early Third Republic. Cambridge: Cambridge University Press, c1993.

NORTH, Michael; ORMROD, David (eds.). Art markets in Europe, 1400-1800. Aldershot; Brookfield: Ashgate, 1998.

SENSIER, Alfred. La Vie et le oeuvre de Jean-François Millet. Manuscrit publié par Paul Mantz avec de nombreuses illustrations, Paris A. Quantin, Imprimeur-editeur, 7, Rue

https://gallica.bnf.fr/ark:/12148/bpt6k96825069.textelmage.

TILBORGH, Louis van. et al. Millet, Van Gogh: Paris Museé d'Orsay Paris: Réunion des musées nationaux: Diffusion, Seuil, 1998. 
felipe sevilhano martinez | mercado de arte e experiência artística no séc. XIX

WHITE, Harrison, WHITE, Cynthia. Canvases and Careers, Institutional Change in The French Painting World. Chicago: University Chicago Press, 1993.

Zarobel, John. Durand-Ruel and the Market for Modern Art, from 1870 to 1873. in "Paul Durand-Ruel and the Modern Art Market: Inventing Impressionism, National Gallery Company, London, Yale University Press, 2015.

Baker, Nienke; Jansen, Leo; Luijten, Hans. Vincent van Gogh: The Letters: The Complete Illustrated and Annotated Edition (Vol. 1-6), Thames \& Hudson: 2009. Disponível em www.vangoghletters.org 